\title{
Nutzen statt Kosten
}

\section{Viele Unternehmen stehen vor der Frage nach dem Nułzen der Einführung eines Umweltmanagementsystems bzw. einer Zertifizierung. Weit verbreitet ist die Meinung, Umweltschutz sei ein reiner Kostenfaktor für die Unternehmen. Den ökonomischen Nułzen hätten einzig die Berater und Zertifizierer. Der Nułzen von Umweltaktivitäten für das Unternehmen wird dabei oft übersehen.}

$\mathrm{M}$

Von Andreas Schlatter it der Norm ISO 14001 'Umweltmanagementsysteme' und dem EG-ÖkoAudit-System steht eine neue Normierungs- und Zertifizierungswelle vor der Tür, die gerade von kleineren und mittleren Unternehmen mit Skepsis betrachtet wird. Die teilweise schlechten Erfahrungen bei der Zertifizierung des Qualitätsmanagementsystems nach ISO 9001 sitzen in vielen Unternehmen noch tief. Hohe Kosten, dicke Ordner voll Papier, aber was ist heute davon noch übrig? Was war der nachhaltige Nutzen der Qualitätsdiskussion im Unternehmen? Ein Umweltmanagementsystem dient einem Unternehmen dazu, systematisch die vom Unternehmen beeinflussbaren Tätigkeiten, Produkte und Dienstleistungen umweltgerechter zu gestalten und diese kontinuierlich zu verbessern (1). Dabei sind die Umweltaktivitäten bezogen auf den langfristigen Nutzen zu betrachten (sustainable advantage), denn Ökologie ist LangzeitÖkonomie (2). In der Praxis wird jedoch häufig nicht der Nutzen betrachtet, sondern einseitig nur die Kosten. Entsprechend werden auch ganze Bücher dem Thema des ökologischen Rechnungswesens und der ökologischen Kostenrechnung gewidmet. Mit einem falsch verstandenem Suchen nach Umweltkosten wird die Grundlage für den Beweis geschaffen, dass Umweltschutz viel kostet und 'nichts bringt'.

Das Ziel einer Kosten- und Nutzenüberlegung ist eine nüchterne Gegenüberstellung von Kosten und Nutzen der Umweltaktivitäten. Dabei dürfen nicht nur rein monetäre Überlegungen angestellt werden; auch die weichen, nichtquantifizierbaren Nutzen sind in die Betrachtung miteinzubeziehen. Als Umweltaktivitäten werden diejenigen Aktivitäten eines Unternehmens bezeichnet, welche auf die Vermeidung und Verminderung negativer Umwelteinwirkungen durch das Unternehmen ausgerichtet sind. Die Umweltaktivitäten eines Unternehmens sind sowohl bezüglich des ökonomischen, als auch des ökologischen Nutzens zu beurteilen.
Dass mit den als 'Umweltaktivitäten' bezeichneten Anstrengungen auch tatsächlich ein Nutzen für die Umwelt, das heisst ein Beitrag zur Vermeidung und Verminderung negativer Umwelteinwirkungen geleistet wird, muss vorausgesetzt werden können. Hierzu schreibt z.B. die Norm ISO 14001 vor, dass ein Unternehmen die bedeutenden Umweltaspekte bestimmen und diese kontinuierlich verbessern soll (3). Die bedeutenden Umweltaspekte sind gerade diejenigen mit den grössten negativen Umwelteinwirkungen. Die nachfolgende Nutzendiskussion konzentriert sich auf den Beitrag der Umweltaktivitäten zur ökonomischen Entwicklung des Unternehmens.

\section{Ökonomischen Nutzen erfassen}

Insgesamt interessiert der ökonomische NettoNutzen, das heisst Nutzen minus Kosten der Umweltaktivitäten. Dabei darf nicht übersehen werden, dass der Nutzen im Gegensatz zu den Kosten meist langfristig anfällt und oft nicht quantifizierbar ist. Zudem sind die Kosten in der betrieblichen Buchhaltung erfassbar, der Nutzen in der Form von Einsparungen und vermiedenen Kosten hingegen nicht.

Die Problematik der Nutzenbetrachtung im Umweltmanagement liegt in der Schwierigkeit, den erzielten Nutzen isoliert erfassen zu können. Das Umweltmanagement stellt einen Bestandteil des gesamten Managements dar und leistet einen Beitrag zur allgemeinen Unternehmensoptimierung. Entsprechend entsteht der Nutzen meist als integrierter Beitrag zu Mehreinnahmen, verminderter Aufwendungen oder vermiedener Opportunitätskosten (4). Die Schwierigkeit liegt weiter darin, allfällige Verbesserungen den vorangegangenen Aktivitäten zuordnen zu können.

Die Evaluation des Nutzens des Umweltmanagements verhält sich somit ähnlich wie der Nutzen der betrieblichen Ausbildung. Nur in ausgewählten Forschungsprojekten konnte bisher der unmittelbare Nutzen der betrieblichen Ausbildung nachgewiesen werden (5). Auch im betrieblichen Umweltschutz ist der Nutzen meist nicht direkt messbar, sondern er ist ein Teil des gesamten Unternehmenserfolgs.

$\mathrm{Zu}$ unterscheiden ist bei der Bestimmung des ökonomischen Nutzens vorab, wovon dieser bestimmt werden soll. Interessante Fragen sind beispielsweise, welcher Nutzen sich aus den aktuellen Umweltaktivitäten des Unternehmens, aus der Stabsstelle 'Umwelt', aus einem einzelnen Umweltprojekt oder auch aus der Zertifizierung des Umweltmanagementsystems für das Unternehmen ergeben. Die Frage nach dem ökonomischen Nutzen des Aufbaus eines Umweltmanagementsystems sollte isoliert hingegen nicht gestellt werden. Ein solches System ist nur eine Systematik für den strukturierten Umgang mit ökologieorientierten Fragestellungen im Unternehmen, die nur so gut ist, wie sie eingerichtet und vor allem angewandt wird. Der Nutzen des Umweltmanagementsystems ergibt sich über die systematische und kontinuierliche Verbesserung der Umweltleistung des Unternehmens.

\section{Vorgehen in fünf Schritten}

Das nachfolgende Vorgehen in fünf Schritten dient der Nutzenbestimmung der Umweltaktivitäten in einem Unternehmen. Je nach der konkreten Fragestellung ist das Vorgehen anzupassen bzw. auch abzukürzen.

Die Idee des Vorgehens ist, mit einer qualitativen Nutzenbeschreibung die Gesamtheit der Nutzen der einzelnen Umweltaktivitäten zu erfassen und anschliessend deren Quantifizierbarkeit zu prüfen. In der abschliessenden Beurteilung des Nettonutzens sind den Kosten die qualitativen und die quantitativen Nutzenaspekte gegenüberzustellen.

1. Systematische Auslegeordnung des Unternehmens mit seinen Tätigkeiten, Produkten und Dienstleistungen

Grundlage für die Nutzenbestimmung von Umweltaktivitäten ist ein fundiertes Systemverständnis der Unternehmensstrukturen sowie der Tätigkeiten, Produkte und Dienstleistungen des Unternehmens.

2. Identifikation der 'Umweltakteure' und ihren 'Umweltaktivitäten'

In einem zweiten Schritt geht es darum, systematisch eine Auslegeordnung der umweltorientierten Aktivitäten und der involvierten 'Umweltakteure' im Unternehmen zu erstellen.

3. Qualitative Nutzenbeschreibung

Für die gezielte und systematische Suche von qualitativen Nutzen der Umweltaktivitäten wurde an der ETH Zürich ein Konzept zur qualitativen Nutzenevaluation entwickelt (4). Die sechs 
übergeordneten Nutzenfelder 'Kosteneffizienz', 'Wettbewerbsfähigkeit', 'Legitimität', 'Legalität', 'Risikoexposition' und 'die Mitgestaltung des ökologischen Strukturwandels' dienen der strukturierten Identifikation der Nutzenpotentiale. Jedes Nutzenfeld ist weiter strukturiert in einzelne Nutzenaspekte (vgl. Abbildung 1).

\section{Prüfen der Quantifizierbarkeit}

Je Nutzenaspekt der einzelnen Umweltaktivitäten ist im vierten Schritt zu prüfen, inwieweit der Nutzen quantifiziert werden kann. Auch semiquantitative Nutzenschätzungen sind in diesem Schritt in Betracht zu ziehen. Semi-quantitativ heisst bestimmen von Grössenordnungen oder von Relationen zu Vergleichsgrössen, etc.

\section{Beurteilen des Nutzens}

Der Nutzen von Umweltaktivitäten setzt sich schliesslich zusammen aus quantifizierten und aus semi-quantitativ bestimmten Nutzenaspekten sowie aus qualitativen Nutzenbeschreibungen. Diese sind gemeinsam den Kosten gegenüberzustellen und zu beurteilen. Das Resultat ist somit kaum eine monetäre Grösse, sondern auch wieder ein Beschrieb des Nettonutzens, respektive ein Nutzen-Argumentarium.

\section{Fazit: Nutzen statt Kosten}

Zusammenfassend kann festgestellt werden, dass durch den Aufbau von Umweltmanagementsystemen, deren Zertifizierung sowie der gézielt durchgeführten Umweltaktivitäten vielfältige ökonomische Nutzen erarbeitet werden können. Mit der Weiterentwicklung von Methoden zur Nutzenbestimmung kann die weit verbreitete Meinung widerlegt werden, dass Umweltschutz nur kostet. Dabei geht es nicht darum, das betriebliche Umweltmanagement $\mathrm{zu}$ Tode $\mathrm{zu}$ quantifizieren, sondern durch eine gesamtheitliche Betrachtung auch nichtquantifizierbare Aspekte in die Beurteilung miteinzubeziehen.

Voraussetzung für die Erzielung von Nutzen durch die Umweltaktivitäten ist ein proaktives Engagement und die Integration ökologischer Betrachtungen in den unternehmerischen Alltag. Werden hingegen Umweltmanagementsysteme als reine Pflichtübungen und die Zertifizierung einzig als Etikette für den Werbeprospekt betrachtet, dann haben nur die Berater und Zertifizierer einen Nutzen und das Unternehmen die entsprechenden Kosten.

\section{Anmerkungen}

(1) Vgl. Schlatter, A.: Umwelt-Dialog - Ökologieorientierte Lernprozesse in Unternehmen. Diss. Nr. 12708, ETH Zürich

\section{Abbildung 1: Modell der Nutzenfelder}

Kosteneffizienz: Unter diesem Titel werden alle direkt quantifizierbaren Nutzenaspekte erfasst. Gezählt werden alle Veränderungen von Stoff- und Energieflüssen, welche einen "Geldstrom" verursachen.

- Wettbewerbsfähigkeit: Die Anstrengungen im Umweltbereich können im Unternehmen Innovationen auslösen, die Marktchancen erhöhen oder weitere Wettbewerbsvorteile bewirken. Diese Vorteile werden in_daher im voraus nur abgeschätzt werden. diesem Nutzenfeld zusammen- Der Nutzen entsteht durch die

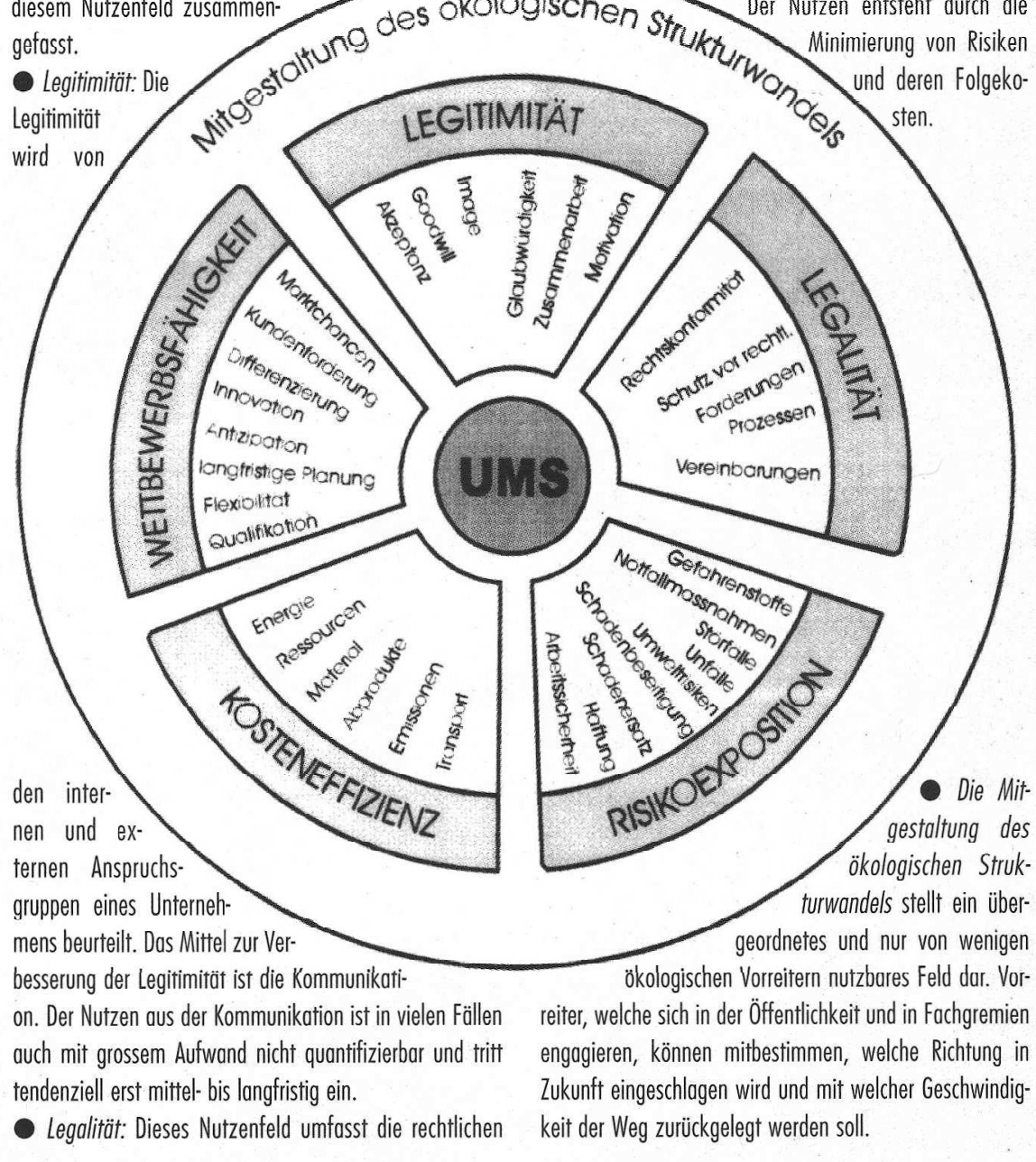

Quelle: Baumgartner, R.: Modell zur Evaluation des unternehmerischen Nutzens eines Umweltmanagementsystems nach ISO 14001. Unveröffentlichte Diplomarbeit, Betriebswissenschaftliches Institut der ETH Zürich 1997.

1998 und Züst, R. : Öko-Performance. Umwelt Focus, Februar 1998, S. 29-31.

(2) Vgl. Becker, C.: Wertorientiertes Umwelt-Management. Diss. Nr. 2101, Universitöt St. Gallen 1998, S. 6 und Gege, M. (Hrsg.): Kosten senken durch Umweltmanagement. 1000 Erfolgsbeispiele aus 100 Unternehmen. Vahlen, München 1997, S. 50.

(3) Vgl. Aichinger, C., G. Coduff, A. Schlatter: Bedeutende Umweltaspekte bestimmen. Qualität und Zuverlässigkeit QZ, Nr. 7/ 1997, S. 781-784.

(4) Vgl. Schlatter, A., R. Baumgartner B. de Quervain, M. Tobler, R. Züst: Business Excellence mit Umweltmanagementsystemen. io Management Nr. 11/ 1997, S. 46-49.

Aspekte, bzw. den Nutzen bei deren konsequenter Einhaltung. Im Zusammenhang mit ISO 14001 oder auch EMAS stellt die Rechtskonformität eine wichtige Voraussetzung zur Erreichung eines Zertifikats dar.

- Risikoexposition: Die Auswirkungen von Risiken sind meist abhängig von Ausfall- oder Eintrittswahrscheinlichkeiten oder von zukünffigen Entwicklungen und können Schen Strukx Munimierung von Risiken

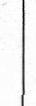


(c) 20I0 Authors; licensee IÖW and oekom verlag. This is an article distributed under the terms of the Creative Commons Attribution Non-Commercial No Derivates License (http://creativecommons.org/licenses/by-nc-nd/3.o/), which permits unrestricted use, distribution, and reproduction in any medium, provided the original work is properly cited. 